\title{
Micromechanical modelling of carbon nanotube reinforced composite materials with a functionally graded interphase
}

\author{
Vahidullah Taç and Ercan Gürses (i)
}

\begin{abstract}
This paper introduces a new method of determining the mechanical properties of carbon nanotube-polymer composites using a multi-inclusion micromechanical model with functionally graded phases. The nanocomposite was divided into four regions of distinct mechanical properties; the carbon nanotube, the interface, the interphase and bulk polymer. The carbon nanotube and the interface were later combined into one effective fiber using a finite element model. The interphase was modelled in a functionally graded manner to reflect the true nature of the portion of the polymer surrounding the carbon nanotube. The three phases of effective fiber, interphase and bulk polymer were then used in the micromechanical model to arrive at the mechanical properties of the nanocomposite. An orientation averaging integration was then applied on the results to better reflect macroscopic response of nanocomposites with randomly oriented nanotubes. The results were compared to other numerical and experimental findings in the literature.
\end{abstract}

\section{Keywords}

Carbon nanotube, polymer, nanocomposite, micromechanics, finite element model, interface, interphase

\section{Introduction}

Discovered in 1991 by Iijima, ${ }^{1}$ carbon nanotubes (CNTs) are the strongest and stiffest materials discovered to date ${ }^{2-6}$ in addition to having outstanding electrical and thermal properties. ${ }^{7}$ While, as of yet, macro-scale use of CNTs in their pristine state remains distant, they are already revolutionizing the materials industry with their role as reinforcements in polymerbased composite materials. ${ }^{8-16}$ There has been a plethora of research articles focusing on the modelling and the determination of the mechanical properties of CNTs since their discovery using a multitude of methodologies ranging from the finite element method, micromechanics and atomistic simulations to experimentations. $7,17,18$ Among these, micromechanics offers quick and easy, yet powerful tools for analysis of nanocomposites.

The vast majority of micromechanical models used for simulating CNT composites are based on the MoriTanaka model ${ }^{19}$ and usually portray the composite using two or three phases, which includes the CNT, matrix (polymer), and the interface. ${ }^{11,12,14,20}$ Latest experimental and molecular mechanics studies of CNT- polymer composites indicate that there are at least four phases in such a composite; the CNT, the interface, the interphase and bulk polymer. ${ }^{8,21,22}$ The term phase refers to semi-ellipsoidal regions of distinct mechanical characteristics in an Eshelby-type micromechanical model. ${ }^{23}$ The interface refers to the thin gap separating the CNT from the polymer and it is dominated by Van der Waals forces in non-functionalized nanocomposites. ${ }^{14,24,25}$ The presence of the CNT modifies the polymer such that it has a higher density near the nanotube, but the density gradually decreases in the radially outward direction from the nanotube longitudinal axis until matching that of bulk polymer. ${ }^{14,21,25}$ This region of variable density is referred to as the "interphase" to distinguish it from the bulk polymer phase which consists of plain polymer with mostly constant mechanical properties and density. The

Department of Aerospace Engineering, Middle East Technical University, Turkey

\section{Corresponding author:}

Ercan Gürses, Department of Aerospace Engineering, Middle East Technical University, Ankara 06800, Turkey.

Email: gurses@metu.edu.tr 
variable nature of the interphase prompts the use of functionally graded phase models in micromechanical modelling of such composites.

In this study, we employed a multi-phase micromechanical model with a functionally graded interphase to accurately model CNT composites. We first identified and characterized the four distinct phases in the composite. We then merged the CNT and interface phases into a single phase that we call the effective fiber. We obtained the properties of this composite phase from finite element analyses. The interphase was modelled in a functionally graded manner using constant, linear and exponential functions for comparison. The outputs of the threephase micromechanical model for a single effective fiber were homogenized using an orientation averaging algorithm and the end results were compared to experimental and computational data available in the literature.

\section{Micromechanical model}

The micromechanical model used in this study is adopted from $\mathrm{Li}^{26}$ and it is a Mori-Tanaka ${ }^{19}$ type multi-inclusion model based on Hori and NematNasser's double-inclusion model. ${ }^{27}$ Like most other self-consistent models, this model relies on two important conclusions reached by J.D. Eshelby in a series of papers. ${ }^{23,28}$ Eshelby concluded that the strain inside an ellipsoidal inclusion embedded in an elastic body is linearly related by the Eshelby tensor to the uniform far field strain in the elastic body, and that this strain is uniform in the inclusion, ${ }^{29}$ i.e.

$$
\langle\varepsilon\rangle_{I}=\boldsymbol{A}\langle\varepsilon\rangle_{V}
$$

where the $\langle\cdot\rangle$ operator shows volume average, subscripts $I$ and $V$ refer to the inclusion and the matrix, and $\boldsymbol{A}$ is a function of the Eshelby tensor $\boldsymbol{S}$. Eshelby tensor can be readily calculated for inclusions that are embedded in an elastic domain. ${ }^{23}$

This principle has been further exploited by Hori and Nemat-Nasser in a recursive formulation to include double inclusions, i.e. inclusions inside other inclusions.

The model posits multiple semi-ellipsoidal elastic phases embedded in an infinite domain to arrive at the mechanical properties of the composite material. The ellipsoids having dimensions $a_{1}, b_{1}, c_{1}$ for the first inclusion, $a_{2}, b_{2}, c_{2}$ for the second inclusion, etc. are assumed to be coaxial, perfectly aligned and of similar shape, so that $\frac{a_{1}}{a_{2}}=\frac{b_{1}}{b_{2}}=\frac{c_{1}}{c_{2}}=\gamma$. A schematic representation of the micromechanical model is given in Figure 1.

The average elastic moduli, $\boldsymbol{C}$, of the composite consisting of $n$ phases are given as

$$
\begin{aligned}
& \boldsymbol{C}=\boldsymbol{C}^{\text {inf }}[\boldsymbol{I}+(\boldsymbol{S}-\boldsymbol{I}) \Lambda][\boldsymbol{I}+\boldsymbol{S} \Lambda]^{-1} \\
& \Lambda=\sum_{i=1}^{n} f_{i} \Lambda_{i}
\end{aligned}
$$

where $\boldsymbol{I}$ is the symmetric fourth-order unity tensor, $\boldsymbol{S}$ is the Eshelby tensor for the ellipsoidal inclusion, ${ }^{23} \boldsymbol{C}^{\text {inf }}$ is the moduli tensor of a fictitious infinite domain. This infinite domain is needed for proper operation of the function. The moduli of this medium were obtained via an iterative process, i.e. some initial guesses were assigned to them and then they were varied until they match the elastic moduli of the resulting composite, $\boldsymbol{C}$. In this study, $n$ is set to either 3 or 4 depending on the assumption of number of phases. $\Lambda$ has to be calculated separately for every phase. If a phase has uniform elasticity throughout, $\Lambda$ is defined as

$$
\Lambda_{i}=\left[\left(\boldsymbol{C}^{i n f}-\boldsymbol{C}_{i}\right)^{-1} \boldsymbol{C}^{i n f}-\boldsymbol{S}\right]^{-1}
$$

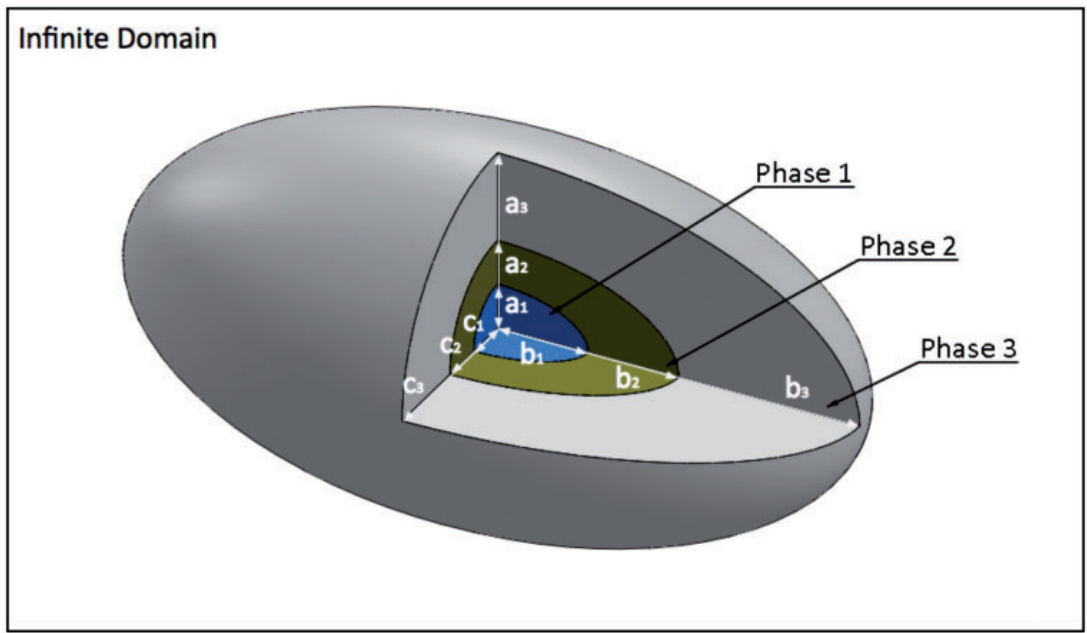

Figure I. Graphical representation of the multi-inclusion micromechanical model. 
Using an integration procedure, this value of $\Lambda$ can be extended to phases of radially varying elasticities by assuming there exist an infinite number of infinitesimally thin ellipsoidal shells that together form one large ellipsoidal phase of varying properties. The integration is shown in $\mathrm{Li}^{26}$ and is reported here

$$
\begin{aligned}
\Lambda_{i} & =\frac{3}{1-\gamma^{3}} \int_{\gamma}^{1} r^{2} \lambda_{i}(r) d r \\
\lambda_{i} & =\left[\left(\boldsymbol{C}^{\text {inf }}-\boldsymbol{C}_{i}(r)\right)^{-1} \boldsymbol{C}^{\text {inf }}-\boldsymbol{S}\right]^{-1}
\end{aligned}
$$

Note that the strain concentration tensor, $\boldsymbol{A}$ and $\Lambda$ represent alternative formulations of the same problem and one can be achieved in terms of the other, as shown in Hori and Nemat-Nasser. ${ }^{27}$

Also note that for a three-phase model specifying $\gamma$ and the volume fraction of the matrix $f_{3}$ also define the volume fractions of the remaining two phases which can be calculated as follows

$$
\begin{aligned}
& f_{1}=\left(1-f_{3}\right) \gamma^{3} \\
& f_{2}=\left(1-f_{3}\right)\left(1-\gamma^{3}\right)
\end{aligned}
$$

\section{Orientation averaging integral}

Orientation averaging integration is an operation used to determine the average value of a tensorial term over all orientations defined by transformation from the local fiber coordinates to global coordinates. The orientation averaging integral of a tensor $\boldsymbol{A}$ is denoted as $<A>$ and is defined as

$$
<A>=\frac{\int_{-\pi}^{\pi} \int_{0}^{\pi} \int_{0}^{\pi / 2} \bar{A}(\phi, \gamma, \psi) g(\phi, \psi) \sin (\gamma) \mathrm{d} \phi \mathrm{d} \gamma \mathrm{d} \psi}{\int_{-\pi}^{\pi} \int_{0}^{\pi} \int_{0}^{\pi / 2} g(\phi, \psi) \sin (\gamma) \mathrm{d} \phi \mathrm{d} \gamma \mathrm{d} \psi}
$$

where

$$
\bar{A}_{i j k l}=c_{i p} c_{j q} c_{k r} c_{l s} A_{p q r s}
$$

$c_{i j}$ are the direction cosines for the transformation and $g$ is the orientation distribution function defined as follows ${ }^{30}$

$$
g(\phi, \psi)=\exp \left(-s_{1} \phi^{2}\right) \exp \left(-s_{2} \psi^{2}\right)
$$

$s_{1}$ and $s_{2}$ are parameters that control the orientation. Three interesting combinations of $s_{1}$ and $s_{2}$ are as follows

\begin{tabular}{lccl}
\hline $\begin{array}{c}\text { Random } \\
\text { orientation }\end{array}$ & $s_{1}=0$ & $s_{2}=0$ & $g(\phi, \psi)=1$ \\
$\begin{array}{c}\text { Aligned } \\
\text { orientation }\end{array}$ & $s_{1}=0$ & $s_{2}=\infty$ & $g(\phi, \psi)=\delta(\phi-0) \delta(\psi-0)$ \\
$\begin{array}{c}\text { Axisymmetric } \\
\text { orientation }\end{array}$ & $s_{1}=k$ & $s_{2}=\infty$ & $g(\phi, \psi)=\exp \left[-k \phi^{2}\right] \delta(\psi-0)$ \\
\hline
\end{tabular}

where $\delta\left(x-x_{0}\right)$ is the Dirac's delta function. In terms of nanocomposites, the three orientations correspond to the cases in which the nanotubes are randomly and evenly distributed, completely aligned in a single direction or partially aligned in a single direction, respectively. The orientation averaging integral was applied to the moduli of the composite material obtained in the previous part using random orientation.

\section{Phases of the nanocomposite CNTs}

The moduli of CNTs are usually obtained through experimental methods such as Raman stereoscopy, 4,6 finite element method, ${ }^{3,9,31}$ or atomistic simulations such as molecular mechanics. ${ }^{5,10,14}$ It is commonly held that CNTs behave in a transversely isotropic manner around the axis of the tube and have a modulus of close to $1 \mathrm{TPa}$ in the longitudinal direction. ${ }^{4-6}$ Tsai et al., ${ }^{14}$ for example use molecular mechanics to obtain the moduli of three single-walled zigzag CNT specimens with radii of $3.9 \AA, 5.5 \AA$ and $7.1 \AA$ and then use energy equivalency methods to replace the CNT with a solid cylinder of similar dimensions. We borrowed their results to be used in our micromechanical model as reported in Table 1.

\section{Interface}

The properties of the interface between CNT and polymer could be computed through atomistic simulations such as molecular mechanics. The interface is an empty space of approximately $3.4 \AA$ thickness dominated by Van der Waals forces but it is sometimes simplified as a

Table I. Mechanical properties of carbon nanotubes.

\begin{tabular}{llllll}
\hline $\begin{array}{l}\text { Radius } \\
\text { [̊] }\end{array}$ & $\begin{array}{l}E_{1} \\
{[\mathrm{GPa}]}\end{array}$ & $\begin{array}{l}G_{12} \\
{[\mathrm{GPa}]}\end{array}$ & $\begin{array}{l}v_{12} \\
{[-]}\end{array}$ & $\begin{array}{l}E_{2} \\
{[\mathrm{GPa}]}\end{array}$ & $\begin{array}{l}v_{23} \\
{[-]}\end{array}$ \\
\hline 3.9 & 1382.5 & 1120 & 0.272 & 645 & 0.2 \\
5.5 & 981.5 & 779.2 & 0.27 & 504 & 0.2 \\
7.1 & 759.9 & 596.3 & 0.27 & 425 & 0.2 \\
\hline
\end{tabular}

Note: Data obtained from Tsai et al. ${ }^{14}$ 
region of isotropic elastic properties. ${ }^{12,14,32}$ The stiffness of the interface depends on many factors like type of polymer, CNT and whether there is chemical functionalization or polymer grafting around the CNT. The interface is expected to be stiff in the presence of functionalization and soft in its absence. To this end, we study the interface using two models; one soft and one stiff. The Young's moduli corresponding to the two models are set at $0.3 E_{B P}$ and $5 E_{B P}$, respectively, while the Poisson's ratio was kept constant at $v_{B P}$, in terms of bulk polymer (BP) moduli. A similar study was previously performed in Wan et al. ${ }^{22}$
Effective fiber

Mori-Tanaka-based micromechanical models assume there are multiple inclusions in the composite in the form of ellipsoids scattered all over the composite ${ }^{33,34}$ as shown in Figure 2(a), while in reality the phases of a nanocomposite are nested as shown in Figure 2(b).

While the model used in this study is originally constructed for nested composites, this is not true for composites where the inclusions have the same aspect ratio as shown in Wang et al. ${ }^{34}$ which is always the case when the phases include the interface and the interphase. This behavior of Mori-Tanaka-based models means that the (a)

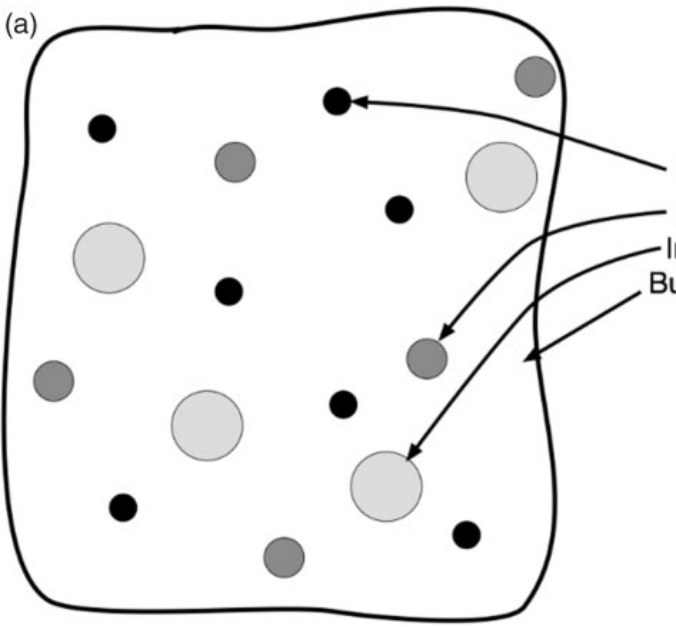

(b)

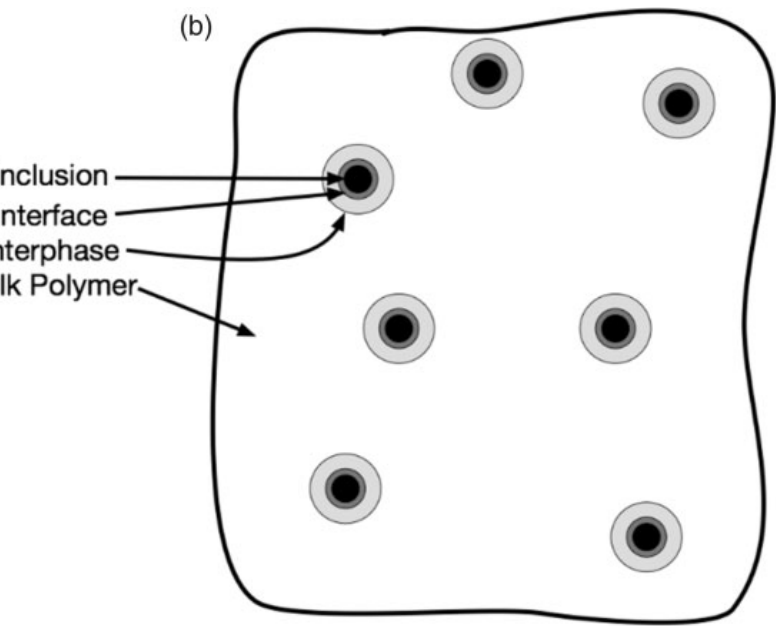

Figure 2. Schematics of micromechanics phase distributions. Mori-Tanaka-based models (a), and annular coated inclusion approach (b).

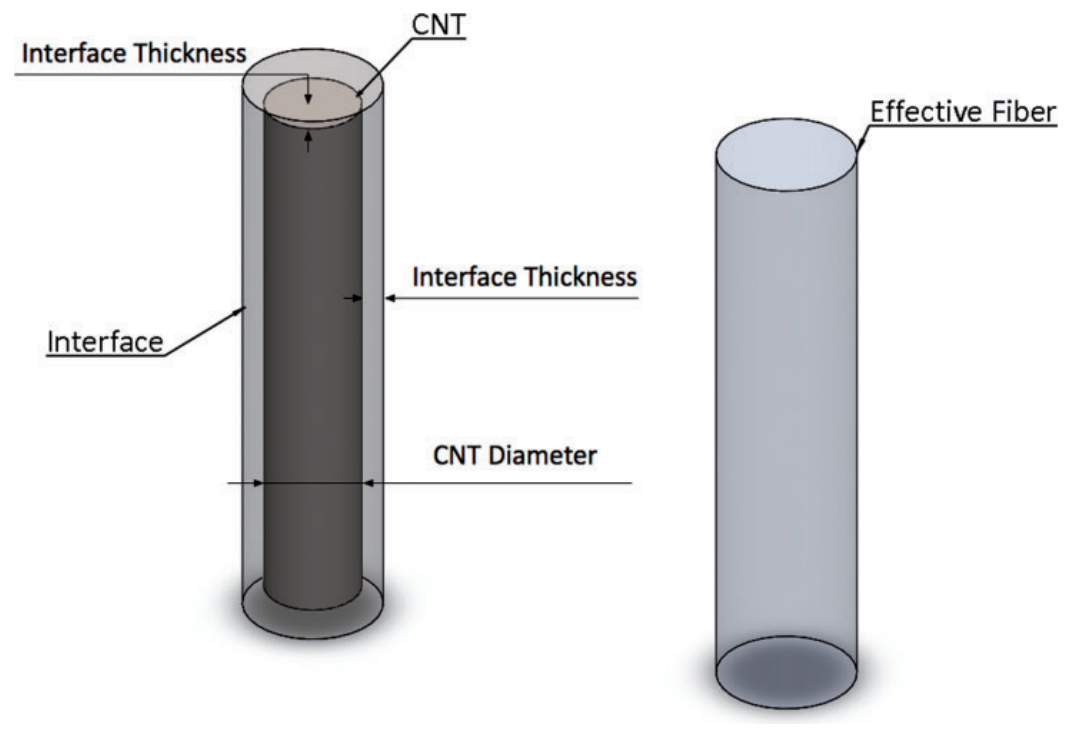

Figure 3. Finite element models consisting of CNT and the interface (left), and effective fiber (right). CNT: carbon nanotube. 
true nature of a nanocomposite cannot be represented accurately using such a model. The phase that is most affected by this is the interface. The greatest impact of the interface in a nanocomposite is through its role in stress transfer from the polymer to the inclusion. However, using the standard Mori-Tanaka approach, this impact is diminished as the inclusion is not coated by the interface. In order to tackle this problem, we combined the CNT and the interface into a single phase that we call the "effective fiber," using finite element modelling. This modeling approach enables accurate representations of roles and contributions of the individual phases to the average elasticity of the nanocomposite. To this end, we constructed two finite element models using Abaqus FEA for each CNT radius as shown in Figure 3. One representing the actual CNT-interface combination consists of two coaxial and concentric cylinders. The inner cylinder represents the CNT and the outer cylinder represents the interface. The inner cylinder is completely submerged within the outer cylinder by an offset corresponding to the thickness of the interface on all sides. The outer surfaces of the inner cylinder and the corresponding inner surfaces of the outer cylinder are then tied to each other assuming a perfect bonding. C3D10 type elements were used with 22,000 elements for the CNT-Interface model and 70,000 elements for the effective fiber model. A mesh size study was performed to make sure the solutions converge. Note that the only parts of the nanocomposite modelled using finite element modelling are the CNT and the interface, while the interphase and the bulk polymer were integrated using the micromechanical model. The dimensions and material properties of the cylinders are the same as those of the CNT and the interface, respectively.

The second finite element model consists of only one cylinder that has the same overall dimensions as the first model. This FE model represents the effective fiber. We assigned a transversely isotropic material and initial guesses to the moduli of the effective fiber model. We then subjected the two models to three loading conditions and measured average displacement at the surfaces of loading. We iteratively changed the moduli of the effective fiber until the displacements of the two models matched, thus obtaining the final elastic moduli of the effective fiber. Note that for some loading scenarios, such as uniform tenion and twisting, there are analytical solutions available in literature for transversely isotropic linear elastic cylinders. Therefore, it is possible to use these analytical solutions for some of the anlayses of the effective anistropic fiber. However, in this work, the analyses of the effective fibers for all three load cases are performed with the finite element method in order to keep the approach also applicable for load cases where there are no analytical solutions available.

Young's modulus $\boldsymbol{E}_{1}$ in the longitudinal direction. To obtain the Young's modulus, we subjected the two FE models to longitudinal compression by applying a pressure on one end of the model and applying a symmetry boundary condition on the other end. Then the average vertical displacement of the top surface of the model was used to adjust the Young's modulus of the effective fiber. This loading scenario is summarized in Figure 4.

Young's modulus $\boldsymbol{E}_{2}$ in the transverse direction and Poisson's ratio $v_{12}$. Transverse Young's modulus and the accompanying Poisson's ratio were obtained by subjecting the two FE models to transverse compression. On one end of the models, a symmetry boundary condition was applied as shown in Figure 5. Then the moduli of the effective fiber were changed iteratively until the average axial and radial displacement components matched those of the CNT-interface FE model.

Shear modulus $\boldsymbol{G}_{12}$ and Poisson's ratio $\nu_{23}$. Out-of-plane shear modulus of the effective fiber required the twisting of the FE models. To this end, we applied a distributed torsional load at one end of the model and a fixed boundary condition at the other end. The average radial displacement of the outermost circle of top surface was then taken as the control variable based on which shear modulus $G_{12}$ of the effective fiber was adjusted. This loading scenario is depicted in Figure 6. In the finite element analyses, it was noted that Poisson's

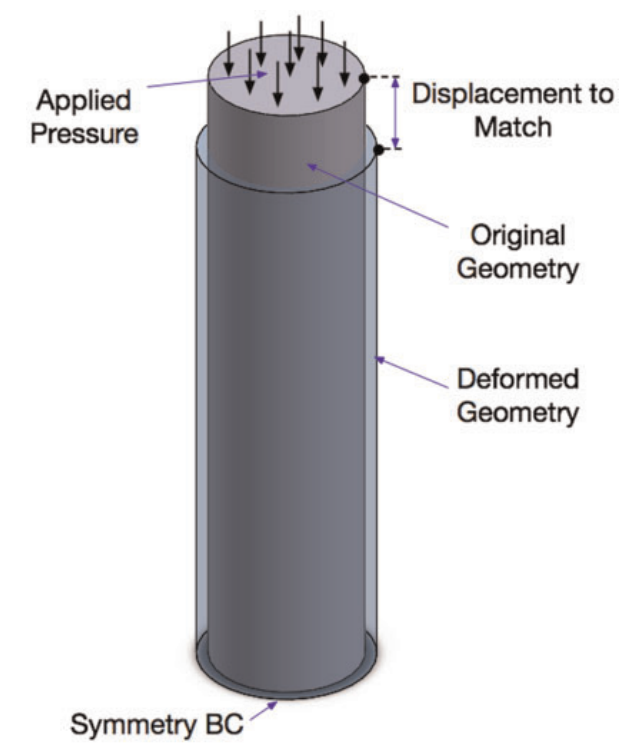

Figure 4. Loading conditions used to obtain the longitudinal modulus of the effective fiber. 


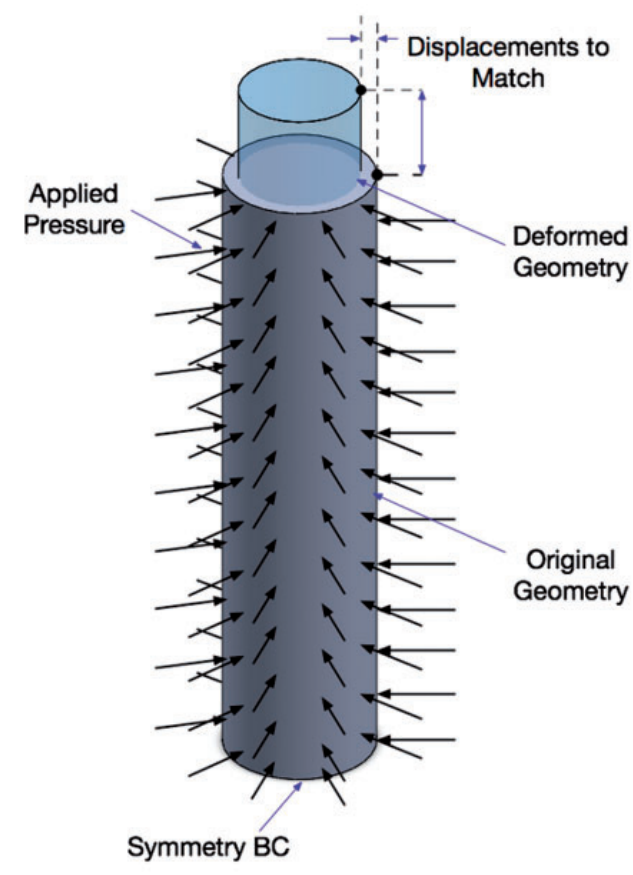

Figure 5. Loading conditions used to obtain the transverse modulus and Poisson's ratio $v_{12}$ of the effective fiber.

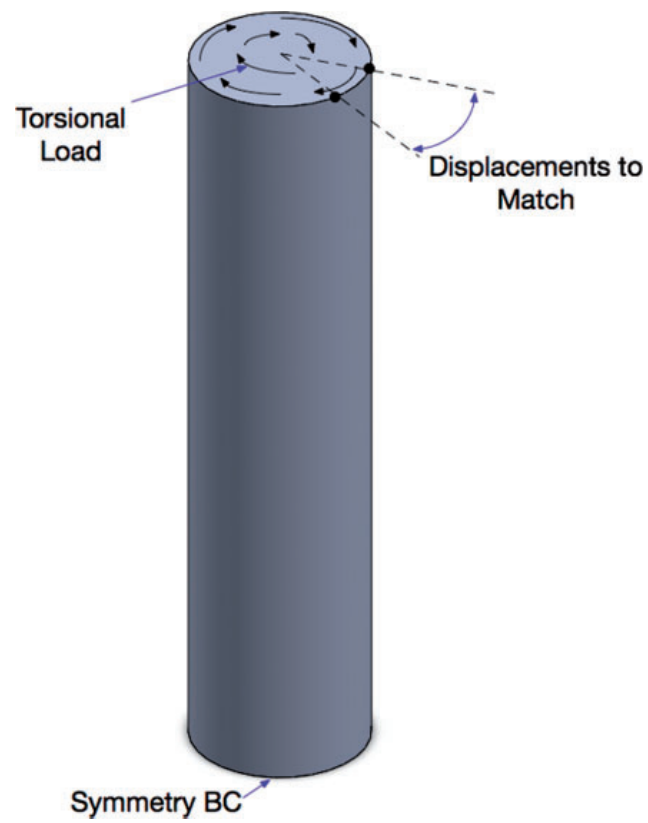

Figure 6. Loading conditions used to obtain $G_{12}$.

ratio $v_{23}$ does not significantly affect the behavior of the model in any of the loading conditions so far. Consequently, we first assumed values of 0.2 and 0.4 for $\nu_{23}$ and observed that the change does not significantly affect the properties of the nanocomposite in the
Table 2. Elastic moduli of the effective fiber using the stiff interface model.

\begin{tabular}{|c|c|c|c|c|c|}
\hline $\begin{array}{l}\mathrm{CNT} \\
\text { radius }[\AA]\end{array}$ & $\begin{array}{l}E_{1} \\
{[\mathrm{GPa}]}\end{array}$ & $\begin{array}{l}E_{2} \\
{[\mathrm{GPa}]}\end{array}$ & $\begin{array}{l}G_{12} \\
{[G P a]}\end{array}$ & $\begin{array}{l}v_{12} \\
{[-]}\end{array}$ & $\begin{array}{l}v_{23} \\
{[-]}\end{array}$ \\
\hline 3.9 & 113.0 & 38.0 & 40.0 & 0.32 & 0.2 \\
\hline 5.5 & 112.0 & 19.6 & 49.0 & 0.32 & 0.2 \\
\hline 7.1 & 73.2 & 17.0 & 39.0 & 0.34 & 0.2 \\
\hline
\end{tabular}

CNT: carbon nanotube.

Table 3. Elastic moduli of the effective fiber using the soft interface model.

\begin{tabular}{|c|c|c|c|c|c|}
\hline $\begin{array}{l}\mathrm{CNT} \\
\text { radius }[\AA]\end{array}$ & $\begin{array}{l}E_{1} \\
{[\mathrm{GPa}]}\end{array}$ & $\begin{array}{l}E_{2} \\
{[\mathrm{GPa}]}\end{array}$ & $\begin{array}{l}G_{12} \\
{[\mathrm{GPa}]}\end{array}$ & $\begin{array}{l}v_{12} \\
{[-]}\end{array}$ & $\begin{array}{l}v_{23} \\
{[-]}\end{array}$ \\
\hline 3.9 & 14.7 & 3.81 & 4.5 & 0.33 & 0.2 \\
\hline 5.5 & 14.0 & 2.01 & 3.8 & 0.61 & 0.2 \\
\hline 7.1 & 13.2 & 1.84 & 2.8 & 0.90 & 0.2 \\
\hline
\end{tabular}

CNT: carbon nanotube.

micromechanical model. Hence, a value of 0.2 was assigned to $v_{23}$ as was done in Tsai et al. ${ }^{14}$

The elastic properties of the effective fiber for the three CNT specimens and two interface models were computed by the procedure explained above. The results are reported in Tables 2 and 3.

Interphase. The interphase is a part of the polymer in the direct vicinity of the CNT that has elevated densities due to the presence of the CNT. It is assumed that the interphase has a location dependent isotropic elastic behavior in which the stiffness starts from a higher value in the inner boundary and decreases in the radially outward direction until reaching the stiffness of the polymer. ${ }^{14,21,35,36}$ We modelled the change using three functions of constant, linear and exponential behavior. The chosen exponential function is as follows

$$
E(x)=\frac{E_{2}-E_{1}}{e^{-\alpha}-1}\left(e^{-\alpha x}-1\right)+E_{1}
$$

where $x=0$ and $E=E_{1}$ at the inner boundary and $x=1, E=E_{2}$ at the outer boundary of the interphase, and $a$ is a parameter that controls the rate of exponential decay. The Young's modulus was assumed to be equal to the longitudinal Young's modulus of the effective fiber at the inner boundary, i.e., $E_{1}=E_{1, e f}$, and that of the polymer at the outer boundary, i.e., $E_{1}=E_{p o l}$, for linearly and exponentially varying models of the interphase. The Poisson's ratio of the interphase was assumed to stay constant. Many highly contrasting values have been reported for the thickness of the interphase from 
$3 \AA$ to $25.5 \AA$; however, there seems to be a general consensus that the thickness of the interphase is independent of the radius of the $\mathrm{CNT}^{21,25,37}$ Interphase and composite Young's modulus distribution is given schematically in Figure 7.

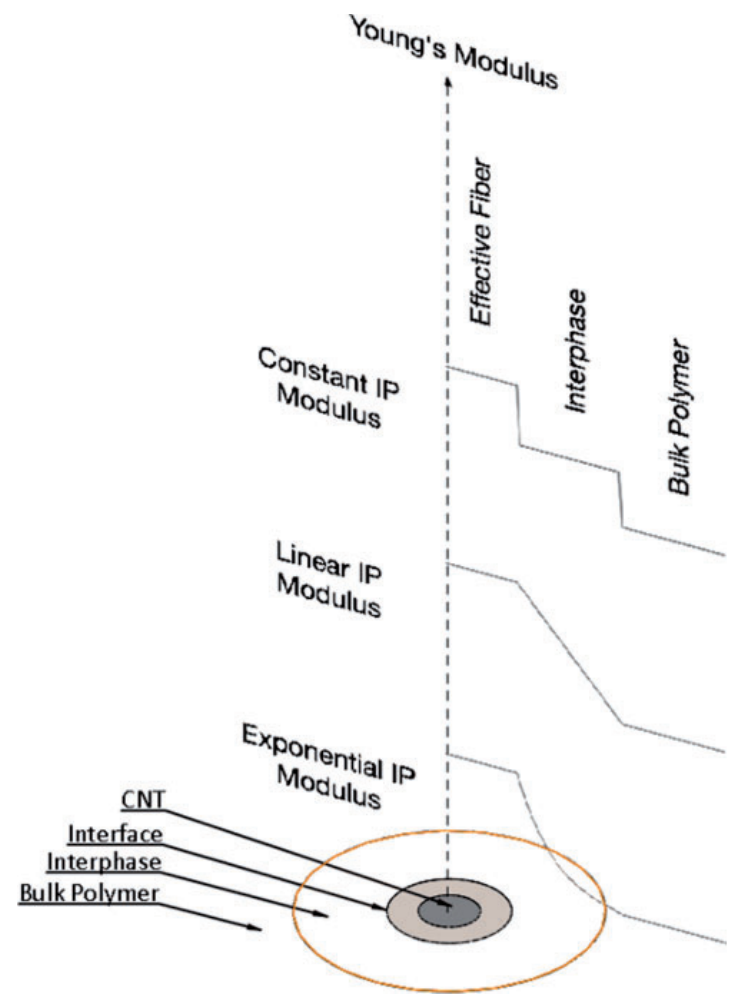

Figure 7. Representative Young's modulus distribution across the micromechanical model.
Bulk polymer. The elastic modulus of the bulk polymer is not very crucial because stiffening effects of the CNT are usually studied relative to base, or polymer modulus. However, selection of a polymer from which nanocomposites have been synthesized and tested enables one to make comparisons with experimental data. As such a polymer named LARC-SI has been adopted which has a Young's modulus of $3.8 \mathrm{GPa}$ and Poisson's ratio of $0.4 .^{8}$

\section{Results and discussion}

The interphase occupies a very large portion of the nanocomposite. It can become the biggest phase in the nanocomposite surpassing even bulk polymer depending on the thickness of the interphase. This means the modulus of the composite heavily depends on the behavior of the interphase. Figure 8 shows how the Young's modulus of the composite increases with respect to that of the polymer when different distributions of interphase modulus are used assuming a moderate interphase thickness of $10 \AA$. A stiff interface model was assumed in this figure. For the constant distribution, the Young's modulus of the interphase was set to the average of the longitudinal Young's modulus of the effective fiber and the Young's modulus of the polymer. It is immediately visible that a constant distribution does not reflect the true nature of the composite, as it appears to cause the stiffening of the composite to increase manyfold, which is not realistic. On the other hand, an exponential distribution of the interphase modulus results in a much more realistic stiffening envelope. While linear distribution also

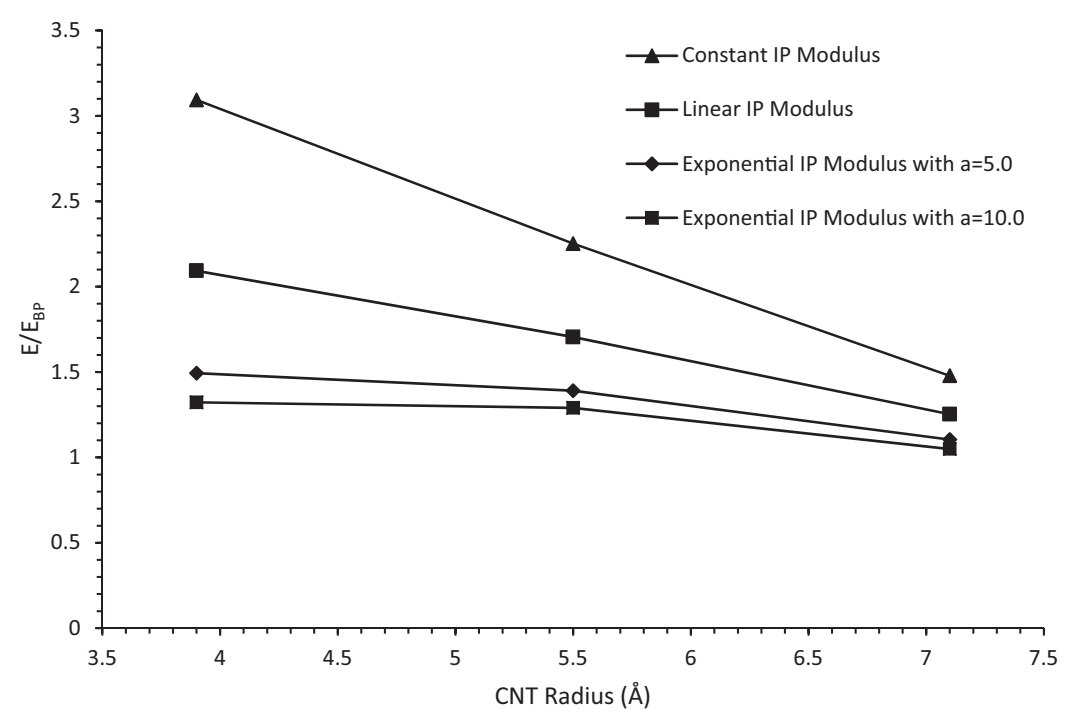

Figure 8. Young's modulus of nanocomposite with \%I vol. effective fiber normalized by that of the polymer vs. CNT radius using various functions for interphase modulus distribution. CNT: carbon nanotube. 
seems to give reasonable results, it lacks the versatility of the exponential distribution with the parameter $a$. The use of an exponential distribution of interphase moduli over the other two alternatives is further motivated by the observations made in the literature ${ }^{14,21,38}$ that suggest the density distribution of the interphase from the interface to the bulk polymer resembles an oscillatory curve with a quasi-exponentially decaying mean. Henceforth, an exponential distribution will be used for the Young's modulus of the interphase.

It appears from Figure 8 that for the same volume fraction the stiffening effect of the CNT diminishes with increasing CNT radius as previously reported in the literature. ${ }^{12,14,39}$ There are two underlying reasons for this. First as a single walled CNT (SWCNT) gets bigger in terms of radius, its effective moduli diminish. Because as the radius grows, so does the empty space inside the CNT. Secondly, as the radius of a CNT grows, the ratio of its surface area to its volume decreases, resulting in decreased contact and adhesion area with the polymer for a given volume fraction.

Figure 9 depicts how the stiffening ratio, $E / E_{B P}$, changes with respect to CNT volume fraction for various CNT radii on a log-lin graph and compares it to some experimental and numerical data available in the literature. ${ }^{8,22,40-47}$ Experimental studies are represented with black markers, while numerical ones are in gray. Interphase thickness, $t_{i p}$, is increased from $3 \AA$ to $5 \AA$
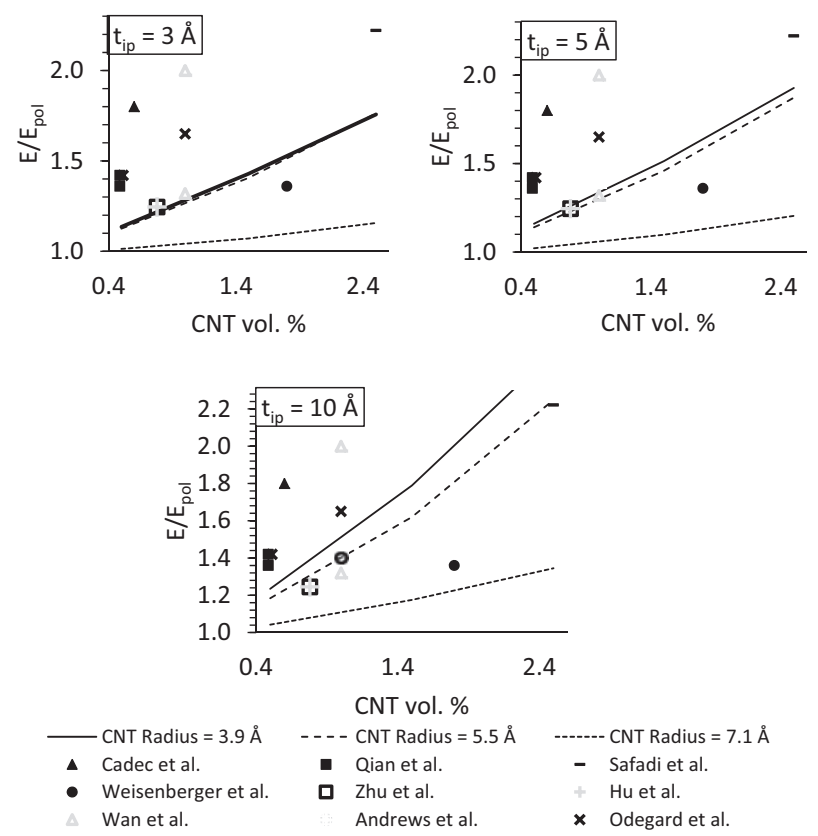

Figure 9. Improvement in polymer Young's modulus vs. CNT vol.\% for various $\mathrm{CNT}$ radii and interphase thicknesses, $\mathrm{t}_{\mathrm{ip}}$, for $\mathrm{a}=\mathrm{I}$. Numerical studies are given in gray markers, while experimental ones are in black. CNT: carbon nanotube. and to $10 \AA$ to observe how interphase thickness affects the results, while parameter $a$ is set at 1 . Note that as the thickness of the interphase increases, the stiffening ratio considerably grows. This is because the interphase is a stronger phase compared to the bulk polymer and hence fulfills a strong role in stiffening of the composite besides the CNT. Increasing the volume fraction of the CNT appears to exponentially increase the stiffening ratio, while at low volume fractions, this behavior is reasonable, and non-dilute effects are expected to start hampering this trend in higher volume fractions. ${ }^{33,39}$ While interphase thicknesses of $3 \AA$ and $5 \AA$ seem to accurately represent some studies found in the literature such as those by Weisenberger et al., ${ }^{44}$ some findings of Wan et al., ${ }^{22} \mathrm{Zhu}$ et al. $^{46}$ and $\mathrm{Hu}$ et al., ${ }^{47}$ it is safe to suggest that most findings can be better represented by an interphase thickness of $10 \AA$.

The analyses so far assumed that the CNTs are randomly distributed in the composite. Such a distribution results in moderate moduli of isotropic nature. However, if there is a nontrivial degree of alignment, the nanocomposite starts to show anisotropic behavior, with a stiffer reaction in some directions and weaker in others. Figure 10 is a plot of Young's modulus (E) in the longitudinal (E1) and transverse (E2) directions as well as shear modulus ( $G, G 12)$, for aligned and random distributions using $a=1$ and $t_{i p}=5 \AA$.

Length of CNTs has a significant effect on the adhesion, and thereby, composite mechanical properties. However, the adhesion properties of the CNT saturate when it reaches a certain length. CNT length is represented in terms of the aspect ratio in the micromechanical model. To study this effect, we set the inclusion shapes such that $a=b$, Aspect Ratio $=\frac{c}{b}=\frac{c}{a}$. As seen in

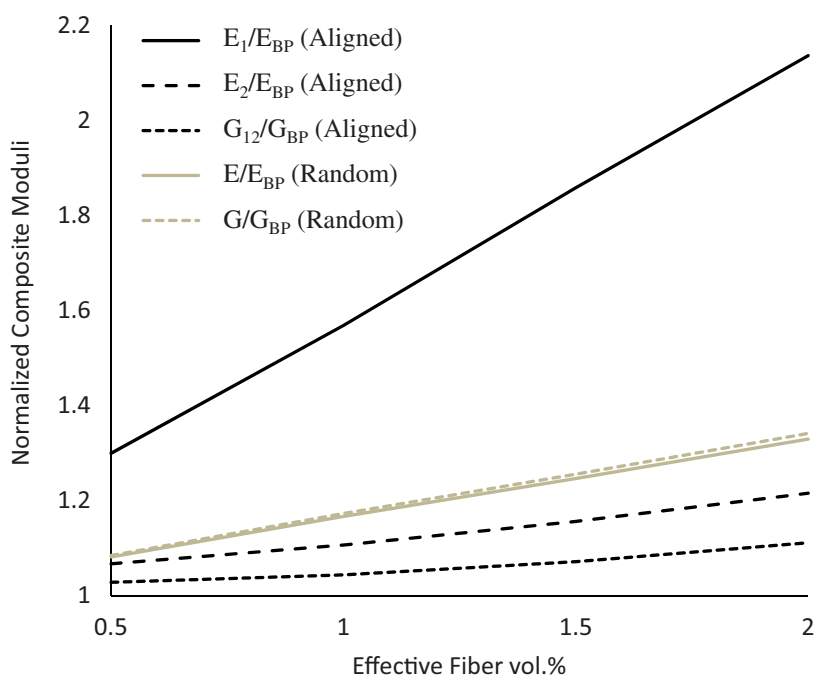

Figure 10. Composite moduli vs. effective fiber vol.\% for aligned and random inclusion distributions. 
the plot of Figure 11, aspect ratio significantly impacts the stiffening effect of the CNT at aspect ratios lower than 20. This is in line with previous observations reported in Ashrafi and Hubert. ${ }^{48}$ This plot was obtained using $a=1, t_{i p}=5 \AA$, a CNT radius of $3.9 \AA$ and $1 \%$ effective fiber by volume.

The contribution of each phase to the stiffness of the nanocomposite was studied in Figures 12 and 13 for the case of stiff and soft interface models. The figures also compare the effective fiber method of this study to a purely micromechanical four-phase model that does not consider the nested quality of the phases. These graphs were obtained by starting from a pure polymer and then introducing the rest of the phases step by step while computing composite stiffness in each step. The figures show three sets of two columns each. The left

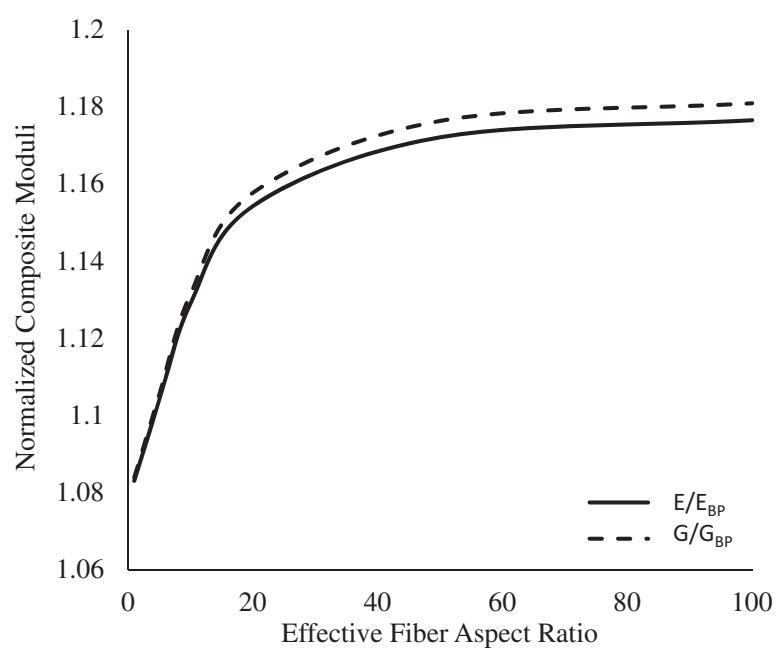

Figure II. Composite Young's and shear moduli vs. effective fiber aspect ratio. column in each set represents the results of the model with an effective fiber characterized by FEM analyses, while the right column is the result of the four-phase purely micromechanical model.

Figure 12 shows that the contribution of the interphase starts from small quantities but rises to match that of the CNT even at an interphase thickness of $10 \AA$. Note that the whole contribution of the interface in this figure is not the area marked with dark gray, but the height difference between the two columns in each set is also caused due to the fact that the interface softens the effective fiber even when using a stiff interface model. This is seen as a justification for the use of the current, effective fiber model.

On the other hand, using a soft interface results in nanocomposites where the contribution of the CNT and the interface is marginal. Instead the interphase becomes the main reinforcement phase in the nanocomposite. However, it should be noted that in this figure the interphase model was not changed between the stiff and soft interface models, as opposed to setting the moduli of the interphase in the inner face equal to those of the effective fiber with soft interface. This is because doing so results in no reinforcement at all. And it is deemed unlikely that the properties of the interface would significantly alter the properties of the interphase. Since the CNT and the interface have little contribution to the stiffness of the nanocomposite, these phases can be omitted from the equation altogether in the case of a soft interface. This is because a soft interface cannot effectively transfer stresses from the polymer to the CNT. In physical terms, this is an indication that two different models should be used for nanocomposite characterization depending on the presence of polymer grafting or functionalization around the CNTs.

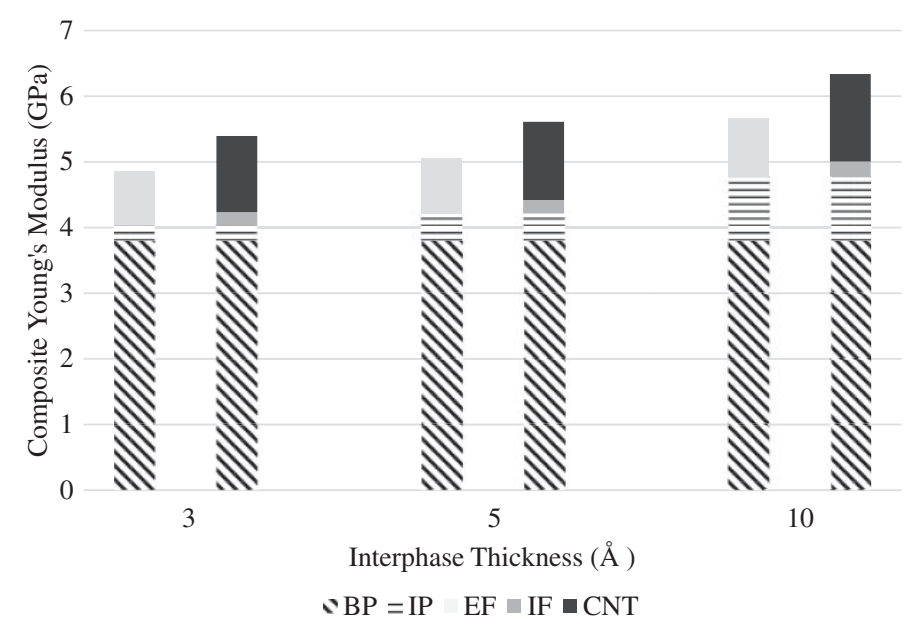

Figure 12. Contribution of each phase to the stiffness of the nanocomposite with a stiff interface. 


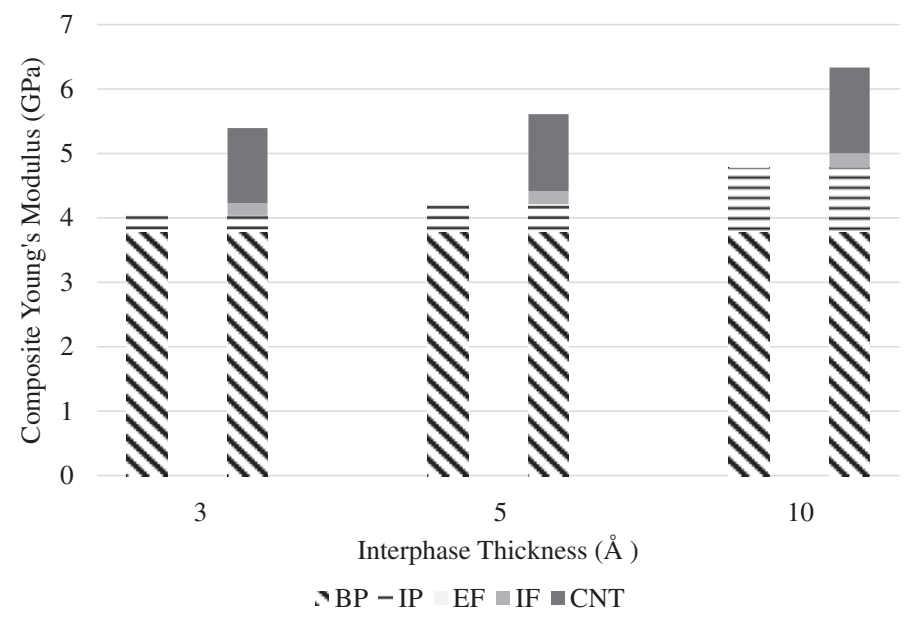

Figure 13. Contribution of each phase to the stiffness of the nanocomposite with a soft interface.

For nanocomposites with polymer grafting or functionalization, the full methodology laid out in this study should be implemented including the FE method for finding effective fiber properties; however, in the case of pristine CNTs, the FE analyses step can be skipped and the CNT and IF phases omitted. Similar findings can be found in the works of Malagù et al. ${ }^{21}$

\section{Conclusions}

An up-close analysis of the constituents of nanocomposites revealed that there are at least four different regions in a nanocomposite that have distinct properties and roles. These are the CNT, the interface, the interphase and the bulk polymer. It was found that the major contribution of the interface comes from its role in stress transfer to the fiber. The interface is the weakest link in the system even assuming other stress transfer mechanisms such as polymer grafting and CNT functionalization are present. This effect was incorporated into our analyses using the finite element method by merging the CNT and interface phases into one. It was also found that, as opposed to the established notion in the literature, the CNT is not the only significant stiffening agent in the nanocomposite. On the contrary, the interphase which is a region of the polymer with modified properties due to the presence of the CNT also plays a key role in stiffening of the composite as reported in Malagù et al. ${ }^{21}$ and Herasati et al. ${ }^{25}$ The study shows that it is important to take the roles and behaviors of the region surrounding the CNT into effect when modeling CNT composites to achieve a high level of fidelity. The three-phase model used in this paper together with an effective fiber obtained through FEM results in composite moduli that are very similar to experimental results found in literature.

\section{Declaration of Conflicting Interests}

The author(s) declared no potential conflicts of interest with respect to the research, authorship, and/or publication of this article.

\section{Funding}

The author(s) received no financial support for the research, authorship, and/or publication of this article.

\section{ORCID iD}

Ercan Gürses (D) https://orcid.org/0000-0001-6030-4063

\section{References}

1. Iijima S. Helical microtubules of graphitic carbon. Nature 1991; 354: 56-58.

2. Lu X and Hu Z. Mechanical property evaluation of singlewalled carbon nanotubes by finite element modeling. Compos Part B Eng 2012; 43: 1902-1913.

3. Kalamkarov AL, Georgiades AV and Rokkam SK. Analytical and numerical techniques to predict carbon nanotubes properties. Int $J$ Solids Struct 2006; 43: 6832-6854.

4. Krishnan A, Dujardin E, Ebbesen TW, et al. Young's modulus of single-walled nanotubes. Phys Rev B 1998; 58: $13-19$.

5. Ávila AF and Lacerda SGR. Molecular mechanics applied to single-walled carbon nanotubes. Mater Res 2008; 11: 325-333.

6. Eric WW, Paul ES and Charles ML. Nanobeam mechanics: elasticity, strength, and toughness of nanorods and nanotubes. Science 1997; 277: 1971-1975.

7. Loos M. Carbon nanotube reinforced composites. 1st ed. Waltham: William Andrew, 2015.

8. Odegard GM, Gates TS, Wise KE, et al. Constitutive modeling of nanotube-reinforced polymer composites. Compos Sci Technol 2003; 63: 1671-1687. 
9. Papanikos P, Nikolopoulos DD and Tserpes KI. Equivalent beams for carbon nanotubes. Comput Mater Sci 2008; 43: 345-352.

10. Yang S, Yu S, Kyoung W, et al. Multiscale modeling of size-dependent elastic properties of carbon nanotube/ polymer nanocomposites with interfacial imperfections. Polymer 2012; 53: 623-633.

11. Yang $\mathrm{S}$ and Cho M. Scale bridging method to characterize mechanical properties of nanoparticle/polymer nanocomposites. Appl Phys Lett 2008; 93: 1-4.

12. Yang $\mathrm{S}$ and Cho M. A scale-bridging method for nanoparticulate polymer nanocomposites and their nondilute concentration effect. Appl Phys Lett 2009; 94: 10-13.

13. Gojny FH, Wichmann MHG, Köpke U, et al. Carbon nanotube-reinforced epoxy-composites: enhanced stiffness and fracture toughness at low nanotube content. Compos Sci Technol 2004; 64: 2363-2371.

14. Tsai JL, Tzeng SH and Chiu YT. Characterizing elastic properties of carbon nanotubes/polyimide nanocomposites using multi-scale simulation. Compos Part B Eng 2010; 41: 106-115.

15. Meng H, Sui GX, Fang PF, et al. Effects of acid- and diamine-modified MWNTs on the mechanical properties and crystallization behavior of polyamide 6. Polymer 2008; 49: 610-620.

16. Ma P-C, Mo S-Y, Tang B-Z, et al. Dispersion, interfacial interaction and re- agglomeration of functionalized carbon nanotubes in epoxy composites. Carbon 2010; 48: 1824-1834.

17. Yuan J, Fan Z, Chen X, et al. Preparation of polystyrene - multiwalled carbon nanotube composites with individual-dispersed nanotubes and strong interfacial adhesion. Polymer 2009; 50: 3285-3291.

18. Velasco-santos C, Martı AL, Ruoff R, et al. Improvement of thermal and mechanical properties of carbon nanotube composites through chemical functionalization. Chem Mater 2003; 15: 4470-4475.

19. Mori $\mathrm{T}$ and Tanaka K. Average stress in matrix and average elastic energy of materials with misfitting inclusions. Acta Metall 1973; 21: 571-574.

20. Klusemann B and Svendsen B. Homogenization methods formulti-phase elastic composites: comparisons and benchmarks. Tech Mech 2010; 30: 374-386.

21. Malagù M, Goudarzi $M$, Lyulin $A$, et al. Diameterdependent elastic properties of carbon nanotube-polymer composites: emergence of size effects from atomistic-scale simulations. Compos Part B Eng 2017; 131: 260-281.

22. Wan H, Delale F and Shen L. Effect of CNT length and CNT-matrix interphase in carbon nanotube (CNT) reinforced composites. Mech Res Commun 2005; 32: 481-489.

23. Eshelby JD. The determination of the elastic field of an ellipsoidal inclusion and related problems. Proc $R$ Soc $A$ 1957; 241: 376-396.

24. Ma PC, Siddiqui NA, Marom G, et al. Dispersion and functionalization of carbon nanotubes for polymer-based nanocomposites: a review. Compos Part A Appl Sci Manuf 2010; 41: 1345-1367.

25. Herasati S, Zhang LC and Ruan HH. A new method for characterizing the interphase regions of carbon nanotube composites. Int J Solids Struct 2014; 51: 1781-1791.
26. Li JY. Thermoelastic behavior of composites with functionally graded interphase: a multi-inclusion model. Int $J$ Solids Struct 2000; 37: 5579-5597.

27. Hori $\mathrm{M}$ and Nemat-Nasser S. Double-inclusion model and overall moduli of multi-phase composites. Mech Mater 1993; 14: 189-206.

28. Eshelby JD. Elastic inclusions and inhomogeneities. In: Sneddon IN and Hill R (eds) Progress in solid mechanics. North Holland, Amsterdam: Interscience, 1962, pp. 89-140.

29. NASA Technical Memorandum TM-103713, 1991, https://ntrs.nasa.gov/search.jsp?R $=19910009919$.

30. Marzari N and Ferrari M. Textural and micromorphological effects on the overall elastic response of macroscopically anisotropic composites. J Appl Mech 1992; 59: 269-275.

31. Tserpes KI and Papanikos P. Finite element modeling of single-walled carbon nanotubes. Compos Part B Eng 2005; 36: 468-477.

32. Wei C, Srivastava D and Cho K. Thermal expansion and diffusion coefficients of carbon nanotube-polymer composites. Nano Lett 2002; 2: 647-650.

33. Wang Z, Oelkers RJ, Lee KC, et al. Annular coated inclusion model and applications for polymer nanocomposites - part I: spherical inclusions. Mech Mater 2016; 101: 170-184.

34. Wang Z, Oelkers RJ, Lee KC, et al. Annular coated inclusion model and applications for polymer nanocomposites - Part II: cylindrical inclusions. Mech Mater 2016; 101: 50-60.

35. Amraei J, Jam JE, Arab B, et al. Modeling the interphase region in carbon nanotube-reinforced polymer nanocomposites. Polym Compos 2019; 40: 1219-1234. DOI: $10.1002 /$ pc. 24950 .

36. Boutaleb S, Zairi F, Mesbah A, et al. Micromechanicsbased modelling of stiffness and yield stress for silica/ polymer nanocomposites. Int J Solids Struct 2009; 46: 1716-1726.

37. Yuan Z, Lu Z, Chen M, et al. Interfacial properties of carboxylic acid functionalized $\mathrm{CNT} /$ polyethylene composites: a molecular dynamics simulation study. Appl Surf Sci 2015; 351: 1043-1052.

38. Choi J, Shin H and Cho M. A multiscale mechanical model for the effective interphase of SWNT/epoxy nanocomposite. Polymer 2016; 89: 159-171.

39. Cho M, Yang S, Chang S, et al. A study on the prediction of the mechanical properties of nanoparticulate composites using the homogenization method with the effective interface concept. Int J Numer Methods Eng 2010; 85: 1564-1583.

40. Cadek M, Coleman JN, Barron V, et al. Morphological and mechanical properties of carbon-nanotubereinforced semicrystalline and amorphous polymer composites. Appl Phys Lett 2002; 81: 5123-5125.

41. Shaffer MSP and Windle AH. Fabrication and characterization of carbon nanotube/poly(vinyl alcohol) composites. Adv Mater 1999; 11: 937-941.

42. Qian D, Dickey EC, Andrews R, et al. Load transfer and deformation mechanisms in carbon nanotube-polystyrene composites. Appl Phys Lett 2000; 76: 2868. 
43. Safadi B, Andrews R and Grulke EA. Multiwalled carbon nanotube polymer composites: synthesis and characterization of thin films. J Appl Polym Sci 2002; 84: 2660-2669.

44. Weisenberger MC, Grulke EA, Jacques D, et al. Enhanced mechanical properties of polyacrylonitrile/ multiwall carbon nanotube composite fibers. $J$ Nanosci Nanotechnol 2003; 3: 535-539.

45. Andrews R, Jacques D, Minot M, et al. Fabrication of carbon multiwall nanotube / polymer composites by shear mixing. Macromol Mater Eng 2002; 287: 395-403.
46. Zhu J, Peng H, Rodriguez-Marcias F, et al. Reinforcing epoxy polymer composites through covalent integration of functionalized nanotubes. Adv Funct Mater 2004; 14 : 643-648.

47. Hu Z, Arefin MRH, Yan X, et al. Mechanical property characterization of carbon nanotube modified polymeric nanocomposites by computer modeling. Compos Part $B$ Eng 2014; 56: 100-108.

48. Ashrafi B and Hubert P. Modeling the elastic properties of carbon nanotube array/polymer composites. Compos Sci Technol 2006; 66: 387-396. 